\title{
On LPI rings
}

\author{
Rachida El Khalfaoui ${ }^{1}$ (D), Najib Mahdou ${ }^{1}$ (D), Abdeslam Mimouni*2 (D) \\ ${ }^{1}$ Department of Mathematics, Faculty of Science and Technology of Fez, Box 2202, University S.M. Ben \\ Abdellah Fez, Morocco \\ ${ }^{2}$ Department of Mathematics and Statistics, King Fahd University of Petroleum \& Minerals, Dhahran \\ 31261, Saudi Arabia
}

\begin{abstract}
In this paper, we extend the $L P I$ property (that is, every locally principal ideal in an integral domain is invertible) to rings with zero-divisors and we study the class of commutative rings in which every regular locally principal ideal is invertible called $L P I$ rings. We investigate the stability of this property under homomorphic image, and its transfer to various contexts of constructions such as direct products, amalgamation of rings and trivial ring extensions. Our results generate examples which enrich the current literature with new and original families of rings that satisfy this property.
\end{abstract}

Mathematics Subject Classification (2010). 13A15, 13F05, 13F10

Keywords. locally principal ideals, regular ideals, trivial extension, pullback, amalgamation of rings, LPI-rings

\section{Introduction}

All rings considered in this paper are assumed to be commutative with identity elements. It is well-known that a finitely generated flat module over a domain is projective, and over an integral domain, the notion of projective ideal is equivalent to the one of invertible ideal. In general, an invertible ideal is projective but the converse is not true. The notion of domains with flat ideals invertible was first studied by Sally and Vasconcelos in 1975 (see [21]) as domains with property $\mathcal{P}$. They showed that if a domain $D$ has the property $\mathcal{P}$, then so does the polynomial ring $D[X]$. In 1977 Glas and Vasconcelos studied the invertibility of faithfully flat ideals over an $\mathrm{H}$-domain and conjectured that over an $\mathrm{H}$ domain, a faithfully flat ideal is finitely generated (and hence invertible) see [14].

In [8], S. Bazzoni conjectured that Prüfer domains for which "an ideal is invertible if and only if it is a locally principal" are exactly the ones with the finite character property, i.e. each nonzero element of the domain belongs to finitely many maximal ideals. This conjecture was first resolved in the affirmative by Holland, Martinez, McGovern and Tesemma ([16]). Later, Halter-Koch stated and proved an analog of Bazzoni's conjecture using the language of ideal systems, that is, $r$-Prüfer monoids, which in the domain case

\footnotetext{
*Corresponding Author.

Email addresses: elkhalfaoui-rachida@outlook.fr (R. El Khalfaoui), mahdou@hotmail.com (N. Mahdou), amimouni@kfupm.edu.sa; a_mimouni@hotmail.com (A. Mimouni)

Received: 31.08.2018; Accepted: 27.03.2019
} 
are $P V M D$ 's and include Prüfer domains ([15]). In 2010, Picozza and Tartarone introduced the notion of quasi-stable ideals as ideals $I$ that are flat in their ring homomorphisms $(I: I)$. They also studied domains in which every ideal is quasi-stable, and proved that Glaz-Vascocncelos conjecture is false ([20]).

In 2011, D. D. Anderson and Muhammad Zafrullah [6] introduced and studied the notion of $L P I$ domains as integral domains in which every nonzero locally principal ideal is invertible. They proved that a finite character intersection of $L P I$ overrings is an $L P I$ domain and so if a domain $D$ is a finite character intersection $D=\cap D_{P}$ for some set of prime ideals of $D$, then $D$ is an LPI domain. In 2013, Kui, Wang and Chen answered positively a question raised by Anderson and Zafrullah of whetter a polynomial ring over an $L P I$ domain is an LPI domain ([17, Theorem 1.8]). In 2014, D. D. Anderson and A. Mimouni studied $L P I$ domains in pullbacks ([3]). Very recently, Xing and Wang F. answered negatively a question by Anderson-Zafurllah by showing that if $R$ is an LPI domain and $S$ is a multiplicatively closed set, then $R_{S}$ need not be an $P L I$ domain (see [22]).

The purpose of the present work is to extend the notion of $L P I$ domain to an arbitrary ring with zero-divisors. A ring $R$ is said to be an $L P I$ ring if every regular locally principal ideal of $R$ is invertible. Noetherian rings are obviously $L P I$ rings by [18, Lemma 18.1]. Our aim is to give some simple methods in order to construct $L P I$ rings outside the context of integral domains that are not Noetherian. For this, we investigate the stability of the LPI property under homomorphic image, and its transfer to various contexts of constructions such as direct products, amalgamation of rings and trivial ring extensions. Our results generate original examples which enrich the current literature with new families of rings satisfying the $L P I$ property. We denote $Z(R)$ the set of zero-divisors of $R$ and by $\operatorname{Reg}(R)$ the set of regular elements in $R$.

Let $A$ be a ring and $E$ an $A$-module. The trivial ring extension of $A$ by $E$ (also called the idealization of $E$ over $A$ ) is the $\operatorname{ring} R=A \ltimes E$ whose underlying group is $A \times E$ with multiplication given by $(a, e)\left(a^{\prime}, e^{\prime}\right)=\left(a a^{\prime}, a e^{\prime}+a^{\prime} e\right)$. Recall that if $I$ is an ideal of $A$ and $E^{\prime}$ is a submodule of $E$ such that $I E \subseteq E^{\prime}$, then $J=I \ltimes E^{\prime}$ is an ideal of $R$. However, prime (resp., maximal) ideals of $\mathrm{R}$ have the form $P \ltimes E$, where $P$ is a prime (resp., maximal) ideal of $A$ [5, Theorem 3.2]. Suitable background on commutative trivial ring extensions is $[5,7,13]$.

Let $A$ and $B$ be two rings, let $J$ be an ideal of $B$ and let $f: A \longrightarrow B$ be a ring homomorphism. In this setting, we consider the following subring of $A \times B$

$$
A \bowtie^{f} J=\{(a, f(a)+j) \mid a \in A, j \in J\}
$$

called the amalgamation of $A$ and $B$ along $J$ with respect to $f$. Moreover, other classical constructions (such as the $A+X B[X], A+X B[[X]]$, and the $D+M$ constructions) can be studied as particular cases of the amalgamation (see [9, Examples 2.5 and 2.6]). A particular case of this construction is the amalgamated duplication of a ring along an ideal $I$ (introduced and studied by D'Anna and Fontana in [9-11]). Let A be a ring, and let I be an ideal of A. $A \bowtie I:=\{(a, a+i): a \in A, i \in I\}$ is called the amalgamated duplication of A along the ideal $I$. See for instance [9-12].

\section{Main results}

Notice that a characterization of locally principal ideals in integral domains is given in [6, Theorem 1]. The following proposition extends this characterization to regular locally principal ideals in rings with zero-divisors. The proof is similar to that one in $[6$, Theorem $1]$, and for the convenience of the reader we include it here. Recall that an ideal $I$ in a ring $R$ is called a cancellation ideal if $I J \subseteq I K$ for ideals $J$ and $K$ of $R$ implies that $J \subseteq K$. 
Proposition 2.1 ([6, Theorem 1$])$. Let $R$ be a ring and let $I$ be a regular ideal of $R$. The following conditions are equivalent:

(1) I is locally principal.

(2) I is faithfully flat.

(3) I is a cancellation ideal.

Proof. (1) $\Rightarrow(2)$ Let $I$ be a regular locally principal ideal of $R$. Then, $I_{M}$ is free for all maximal ideals $M$ of $R$. Indeed, $I_{M}$ is a regular ideal of $R_{M}$ for all $M \in \operatorname{Max}(R)$ since $I$ is regular. Therefore, $I_{M}$ is a regular principal ideal of $R_{M}$ for all $M \in \operatorname{Max}(R)$ and so $I_{M}$ is genereted by a regular element and so it is free. Hence, $I_{M}$ is faithfully flat for all $M \in \operatorname{Max}(R)$. Then, $I$ is faithfully flat.

$(2) \Rightarrow(3)$ Let $I$ be a regular faithfully flat ideal of $R$. Let $I J \subseteq I K$ for some ideals $J$ and $K$ of $R$. We concider the exact sequence,

$$
0 \rightarrow K \rightarrow K+J \rightarrow \frac{K+J}{K} \rightarrow 0 .
$$

Since $I$ is faithfully flat, then we have

$$
0 \rightarrow I \otimes K \rightarrow I \otimes(K+J) \rightarrow I \otimes \frac{K+J}{K} \rightarrow 0 .
$$

Therefore, $I \otimes \frac{K+J}{K}=\frac{I \otimes(K+J)}{I \otimes K}=\frac{I(K+J)}{I K}=\frac{I K}{I K}=0$. Since $I$ is faithfully flat, then $\frac{K+J}{K}=0$ and so $J \subseteq K$.

$(3) \Leftrightarrow(1)$ By $[4$, Theorem].

Next, we study the transfer of the $L P I$ property to direct products.

Theorem 2.2. Let $\left(R_{i}\right)_{i=1, \cdots, n}$ be a family of commutative rings. Then $R=\prod_{i=1}^{n} R_{i}$ is an LPI ring if and only if so is $R_{i}$ for each $i=1, \cdots, n$.

The proof of this theorem needs the following lemmas. The proof of the first lemma is straightforward and it is omitted, and the second lemma is a well-known result.

Lemma 2.3. Let $\left(R_{i}\right)_{i=1,2}$ be two rings and $I_{i}$ be an ideal of $R_{i}$ for $i=1,2$. Then, $I_{1} \times I_{2}$ is a regular locally principal ideal of $R_{1} \times R_{2}$ if and only if $I_{1}$ and $I_{2}$ are regular locally principal ideals of $R_{1}$ respectively $R_{2}$.

Lemma 2.4 ([18, Lemma 18.1]). Let I be a regular locally principal ideal of a ring $A$. Then, $I$ is invertible if and only if it is finitely generated.

Proof of Theorem 2.2. The proof is done by induction on $n$ and it suffices to check it for $n=2$. By Lemma 2.3, $I_{1} \times I_{2}$ is a regular locally principal ideal of $R_{1} \times R_{2}$ if and only if $I_{1}$ and $I_{2}$ are locally principal ideals of $R_{1}$ respectively $R_{2}$ and it is easy to see that $I_{1} \times I_{2}$ is a finitely generated ideal of $R_{1} \times R_{2}$ if and only if $I_{1}$ and $I_{2}$ are finitely generated ideals of $R_{1}$ respectively $R_{2}$ and we conclude by Lemma 2.4.

The next result shows that the $L P I$-property descends into a faithfully flat ring homomorphism.

Proposition 2.5. Let $R$ and $S$ be two rings and $f: R \rightarrow S$ be a ring homomorphism making $S$ a faithfully flat $R$-module. Assume that $f(\operatorname{Reg}(R)) \subseteq \operatorname{Reg}(S)$. If $S$ is an LPI ring, then so is $R$.

In particular, if $R$ and $S$ are two domains and $f: R \rightarrow S$ is a ring homomorphism making $S$ a faithfully flat $R$-module then if $S$ is an LPI domain, then so is $R$.

Proof. Let $I$ be a regular locally principal ideal of $R$. Then $I$ is a faithfully flat $R$-module by Proposition 2.1. So $I \otimes S=I S$ is a faithfully flat $S$-module. Since $f(\operatorname{Reg}(R)) \subseteq \operatorname{Reg}(S)$, $I S$ is a regular locally principal ideal of $S$ and so it is invertible since $S$ is an $L P I$-ring which, in turn, is equivalent to $I S$ is finitely generated. Therefore $I \otimes S=I S$ is finitely generated. Hence $I$ is a finitely generated ideal of $R$ (as $S$ is a faithfully flat $R$-module), and therefore $I$ is an invertible ideal of $R$. Thus $R$ is an $L P I$ ring as desired. 
As an immediate consequence, we recover [6, Teorem 5 (1)].

Corollary 2.6 ([6, Theorem $5(1)])$. Let $R$ be a domain and let $X$ be an indeterminate over $R$. If $R[X]$ (resp.R $[[X]]$ ) is an LPI ring, then so is $R$.

Now, we will see the transfer of the $L P I$ property to homomorphic image. Recall that an ideal $I$ of a ring $R$ is a pure ideal if for every maximal ideal $M \in \operatorname{Max}(R), I_{M}=0_{M}$ or $I_{M}=R_{M}$.

Proposition 2.7. Let $R$ be a ring.

(1) Let $P$ be a finitely generated non-regular prime ideal of $R$ such that $P$ is contained in all regular locally principal ideals of $R$. If $\frac{R}{P}$ is an LPI ring, then so is $R$.

(2) Let $I$ be a regular pure ideal of $R$. If $R$ is an LPI ring, then $\frac{R}{I}$ is an LPI ring.

The proof of this proposition needs the following lemma.

Lemma 2.8. Let $P$ be a non-regular prime ideal of a ring $R$.

If $J$ is a regular locally principal ideal of $R$ such that $P \subseteq J$, then $\frac{J}{P}$ is a regular locally principal ideal of $\frac{R}{I}$.

Proof. Let $J$ be a regular locally principal ideal of $R$ such that $P \subseteq J$ and let $a$ be a regular element of $J$. Necessarily $a \notin P$. Now, for every $x \in R, \overline{a x}=\overline{\overline{0}}$ in $\frac{J}{P}$ implies that $a x \in P$. Hence $x \in P$ since $P$ is prime. Then, $\bar{x}=0$ and so $\bar{a}$ is a regular element in $\frac{J}{P}$. Therefore, $\frac{J}{P}$ is a regular ideal of $\frac{R}{I}$. Thus if $J$ is locally principal, then the localization of $\frac{J}{P}$ at any maximal ideal $\frac{M}{P}$ of $\frac{R}{P}$ is isomorphic to $\frac{J_{M}}{P_{M}}$. Since $J_{M}$ is principal, $\frac{J_{M}}{P_{M}}$ is principal and therefore $\frac{J}{P}$ is locally principal.

Proof of Proposition 2.7. (1) Let $P$ be a finitely generated non-regular prime ideal of $R$ contained in all regular locally principal ideals. Assume that $\frac{R}{P}$ is an LPI ring. Let $J$ be a regular locally principal ideal of $R$. Then $\frac{J}{P}$ is a regular locally principal ideal of $\frac{R}{P}$ (Lemma 2.8) and so it is invertible. By Lemma 2.4, $\frac{J}{P}$ is finitely generated. Now, by the exact sequence,

$$
0 \longrightarrow P \longrightarrow J \longrightarrow \frac{J}{P} \longrightarrow 0
$$

$J$ is finitely generated and by Lemma 2.4 we conclude that $J$ is invertible. Hence $R$ is an $L P I$ ring.

(2) Let $\frac{J}{I}$ be a regular locally principal ideal of $\frac{R}{I}$. Then $\frac{J}{I}$ is a faithfully flat $\frac{R}{I}$-module. Since $I$ is a regular ideal of $R$ and $I \subseteq J$, then $J$ is a regular ideal of $R$. Now, consider the following exact sequence:

$$
0 \rightarrow I \rightarrow J \rightarrow \frac{J}{I} \rightarrow 0
$$

Since $I$ is a regular pure ideal of $R, I$ is a regular locally principal ideal of $R$. So $I$ is a faithfully flat ideal of $R$ by Proposition 2.1. Thus $\frac{J}{I}$ is a flat $R$-module (since $\frac{R}{I}$ is a flat $R$-module). By the exact sequence, we conclude that $J$ is a faithfully flat ideal of $R$. Hence $J$ is a regular locally principal ideal of $R$. So $J$ is invertible and so finitely generated. Therefore $J \otimes \frac{R}{I}=\frac{J}{I}$ is a finitely generated ideal of $\frac{R}{I}$ and so it is invertible by Lemma 2.4. Therefore $\frac{R}{I}$ is an $L P I$ ring.

Example 2.9. Let $K$ be a field and $R=K \ltimes K$ and let $P=0 \ltimes K$. Then $P$ is a finitely generated non-regular prime ideal of $R$ that is contained in all regular ideals of $R$ by Lemma 2.12. Since $\frac{R}{P}=\frac{K \ltimes K}{0 \ltimes K}=K, K \ltimes K$ is an $L P I$ ring by Proposition 2.7 .

Our next theorem develops a result on the transfer of the $L P I$ property to trivial ring extension. Recall that if $E$ is an $A$-module, then $Z(E)=\{a \in A$ such that $a e=0$ for some $0 \neq e \in E\}$. 
Theorem 2.10. Let $A$ be a ring, $E$ an $A$-module, $R=A \ltimes E$ the trivial ring extension of $A$ by $E$ and let $S=A \backslash(Z(A) \cup Z(E))$.

(1) Assume that for every regular locally principal ideal $I$ of $A, I \cap S \neq \emptyset$. If $R$ is an $L P I$ ring, then so is $A$.

(2) Assume that $E$ is torsion-free and divisible. Then $A$ is an LPI ring if and only if so is $R$. In particular, if $A$ is a domain and $E$ is a flat divisible $R$-module, then $A$ is an LPI ring if and only if so is $R$.

(3) Assume $E$ is finitely generated and $E=S^{-1} E$. If $A$ is an LPI ring, then so is $R$. If furthermore, for every regular locally principal ideal $I$ of $A, I \cap S \neq \emptyset$, then $A$ is an LPI ring if and only if so is $R$.

The proof of this theorem needs the following lemmas.

Lemma 2.11. (1) Let $A$ be a ring, $E$ an $A$-module and $I$ an ideal of $A$ and let $S=$ $A \backslash(Z(A) \cup Z(E))$.

(a) If $I \ltimes I E$ is a regular locally principal ideal of $A \ltimes E$, then $I$ is a regular locally principal ideal of $A$.

(b) If $I \cap S \neq \emptyset$, then $I$ is a regular locally principal ideal of $A$ if and only if $I \ltimes I E$ is a regular locally principal ideal of $A \ltimes E$.

(c) If $E$ is torsion free, then $I$ is a regular locally principal ideal of $A$ if and only if $I \ltimes I E$ is a regular locally principal ideal of $A \ltimes E$.

(2) Let $E^{\prime}$ is a submodule of $E$. If $I \ltimes E^{\prime}$ is a regular locally principal ideal of $A \ltimes E$, then so is $I$.

Proof. (1) (a) Clearly if $(a, e)$ is a regular element of $I \ltimes I E$, then $a$ is a regular element of $I$ by [1, Lemma 6]; and $I$ is a locally principal ideal of $A$ if and only if $I \ltimes I E$ is a locally principal ideal of $A \ltimes E$ by [1, Theorem 7].

(b) Assume that $I \cap S \neq \emptyset$. Then there exists a regular element $a$ in $I$ such that $a \notin Z(E)$. Clearly $(a, 0)$ is a regular element of $I \ltimes I E$. Indeed, let $(0,0) \neq(b, e) \in I \ltimes I E$ such that $(a, 0)(b, e)=(0,0)$. Then $a b=0$ and $a e=0$. Since $a$ is regular, $b=0$ and since $a \notin Z(E)$, $e=0$. Thus $(b, e)=(0,0)$ and so $(a, 0)$ is regular. Hence $I \ltimes I E$ is a regular ideal of $A \ltimes E$.

(c) Follows from [2, Lemma 10].

(2) Let $E^{\prime}$ is a submodule of $E$ such that $I \ltimes E^{\prime}$ is a regular locally principal ideal of $A \ltimes E$. Then $I$ is a regular ideal of $A$ and for every maximal ideal $M$ of $R$,

$$
\begin{aligned}
\left(I \ltimes E^{\prime}\right)_{M \ltimes E} & =I_{M} \ltimes E_{M} \\
& =\left(A_{M} \ltimes E_{M}\right)(x, e) \\
& =A_{M} x \ltimes\left(A_{M} e+E_{M} e\right)
\end{aligned}
$$

Hence $I_{M}=A_{M} x$ and therefore $I$ is a locally principal ideal of $A$.

Lemma 2.12 ([5, Theorem 3.9]). Let $A$ be a ring, $E$ an $A$-module and $S=A \backslash(Z(A) \cup$ $Z(E))$. Then the following conditions are equivalent.

(1) Every regular ideal of $A \ltimes E$ has the form $I \ltimes E$ where $I$ is an ideal of $A$ with $I \cap S \neq \emptyset$.

(2) Every regular ideal of $A \ltimes E$ is homogenous.

(3) $E=S^{-1} E$.

Lemma 2.13 ([1, Theorem 7]). Let $A$ be a ring, $I$ a nonzero ideal of $A$ and $E$ an $A$ module. If $I \ltimes I E$ is an invertible ideal of $A \ltimes E$, then $I$ is invertible.

Lemma 2.14 ([2, Theorem 11]). Let $A$ be a ring, $E$ be a torsion-free and divisible $A$ module and $I \ltimes N$ a homogenous ideal of $A \ltimes E$. If $I$ is an invertible ideal of $A$, then $I \ltimes N$ is invertible. 
Lemma 2.15 ([1, Theorem 9]). Let $A$ be a ring, $E$ an $A$-module, $I \ltimes N$ an homogeneous ideal of $A \ltimes E$. If I is a finitely generated ideal of $A$ and $N$ is a finitely generated submodule of $E$, then $I \ltimes N$ is finitely generated.

Proof of Theorem 2.10. (1) Assume that for every regular locally principal ideal $I$ of $A, I \cap S \neq \emptyset$ and let $I$ be a regular locally principal ideal of $A$. By Lemma 2.11 (1)(b), $I \ltimes I E$ is a regular locally principal ideal of $A \ltimes E$. Since $A \ltimes E$ is an $L P I$ ring, then $I \ltimes I E$ is invertible. By Lemma 2.13, $I$ is invertible and so $A$ is an $L P I$ ring.

(2) Assume that $E$ is torsion-free and divisible. Then $E=S^{-1} E$ where $S=A \backslash(Z(A) \cup$ $Z(E)$ ) (since $E$ is divisible).

Let $J$ be a regular locally principal ideal of $R$. By Lemma 2.12, $J$ has the form $I \ltimes E$ where $I$ is an ideal of $A$ with $I \cap S \neq \emptyset$. Thus $I$ is a regular locally principal ideal of $A$ by Lemma 2.11 (2). Since $A$ is an $L P I$ ring, $I$ is invertible. By Lemma $2.14, I \ltimes E$ is an invertible ideal of $A \ltimes E$ and therefore $A \ltimes E$ is an $L P I$ ring.

Conversely, assume that $A \ltimes E$ is an $L P I$ ring. Since $E$ is torsion free, $Z(E) \subseteq Z(A)$ and so for every regular locally principal ideal $I$ of $A, I \cap S \neq \emptyset$. Hence $A$ is an $L P I$ ring by (1).

(3) Assume that $E$ is finitely generated, $E=S^{-1} E$ and $A$ is an $L P I$ ring. Let $I \ltimes E$ be a regular locally principal ideal of $R$ where $I$ is an ideal of $A$ with $I \cap S \neq \emptyset$. Then $I$ is a regular locally principal ideal of $A$ (Lemma $2.11(2)$ ) and thus $I$ is invertible (and so is finitely generated). Since $E$ is finitely generated, $I \ltimes E$ is a finitely generated ideal of $A \ltimes E$ (Lemma 2.15) and therefore it is invertible by Lemma 2.4. Thus $A \ltimes E$ is an $L P I$ ring. If furthermore, for every regular locally principal ideal $I$ of $A, I \cap S \neq \emptyset$, then the equivalence by (1).

Theorem 2.10 leads to the following result.

Corollary 2.16. Let $A$ is a domain and $E$ a $K$-vector space where $K=q f(A)$. Then $A \ltimes E$ is an LPI ring if and only if so is $A$.

Example 2.17. Let $A=\mathbb{Z}_{(2)}=\left\{\frac{a}{b} \mid a, b \in \mathbb{Z}\right.$ and $b$ is not divisible by 2$\}$ and $E=\frac{\mathbb{Q}}{\mathbb{Z}_{(2)}}$. Then $A \ltimes E$ is an $L P I$ ring.

Proof. $\mathbb{Z}_{(2)}$, as a $D V R$, is an $L P I$ ring.

$\frac{\mathbb{Q}}{\mathbb{Z}_{(2)}}$ is a divisible $\left(\mathbb{Z}_{(2)}\right)$-module since $Q$ is a divisible $\left(\mathbb{Z}_{(2)}\right)$-module. Also, $\frac{\mathbb{Q}}{\mathbb{Z}_{(2)}}=\left\{\frac{a}{b} \mid\right.$ $a, b \in \mathbb{Z}$ and $b$ is divisible by 2$\}$ is a flat $\left(\mathbb{Z}_{(2)}\right)$-module since it is a free module generated by $\frac{1}{2}$. So $A \ltimes E$ is an $L P$-ring by Theorem 2.10 (2). ian.

By Theorem 2.10, we are able to give new examples of $L P I$ rings that are not Noether-

Example 2.18. Let $K$ be a field and $E$ be an infinite dimensional vector space over $K$. Then :

(1) $K \ltimes E$ is an $L P I$ ring (by Corollary 2.16).

(2) $K \ltimes E$ is not Noetherian (since $E$ is not finitely generated and by [5, Theorem $4.8])$.

Next, we will see the transfer of the $L P I$ property to the amalgamated duplication. Recall that an ideal $J$ of $A \bowtie I$ is called homogeneous if $J=K \bowtie I$ for some ideal $K$ of A. If $K$ is an ideal of $A \bowtie I$ such that $0 \times I \subseteq K$, then $K$ is homogeneous (see [19, Lemma $2.9])$. 
Theorem 2.19. Let $A$ be a ring and $I$ be an ideal of $A$.

(1) Assume that I is flat. If $A \bowtie I$ is an LPI ring, then so is $A$.

(2) Assume that $I$ is a non-regular finitely generated ideal of $A$ and $S^{-1} I=I$ where $S=A \backslash Z(A)$. If $A$ is an LPI ring, then so is $A \bowtie I$. If furthermore $I$ is flat, then $A$ is an LPI ring if and only if so is $A \bowtie I$.

The proof of this theorem needs the following lemmas. The proofs of the first and third lemma are elementary proofs and for convenience we include them here.

Lemma 2.20. Let $A$ be a ring and $I$ be an ideal of $A$. If $(a, a+i)$ is a regular element of $A \bowtie I$, then $a$ is a regular element of $A$.

Proof. Let $(a, a+i)$ be a regular element of $A \bowtie I$ and $b \in A$ such $a b=0$. If there exists $j \in I$ such that $j b \neq 0$, then $(a, a+i)(b j, 0)=(0,0)$, which is absurd since $(a, a+i)$ is regular. Hence, for all $j \in I, j b=0$. Thus $(a, a+i)(b, b)=(0,0)$ and so $(b, b)=(0,0)$. Hence $a$ is a regular element of $A$.

Lemma 2.21. Assume that $I$ is a non-regular proper ideal of a ring $A$ and $S=A \backslash(Z(A))$. The following are equivalent:

(1) All regular ideals of $A \bowtie I$ are homogeneous.

(2) For all $a \in S$ we have $I=a I$ which is equivalent to $I=S^{-1} I$.

Proof. (1) $\Rightarrow(2)$ Assume that all regular ideals of $A \bowtie I$ are homogeneous and let $a \in S$. Then $(a, a)$ is a regular element of $A \bowtie I$ and so $\langle(a, a)\rangle$ is a homogeneous ideal of $A \bowtie I$. Thus $0 \times I \subseteq\langle(a, a)\rangle=J \bowtie I$ for some ideal $J$ of $A$. Let $i \in I$. Then, $(0, i)=(\alpha, \alpha+k)(a, a)$ where $\alpha \in A$ and $k \in I$. Since $a$ is a regular element of $A, \alpha a=0$ implies that $\alpha=0$. So $(0, i)=(0, k)(a, a)$. Thus $i=k a$ and so $I=a I$.

$(2) \Rightarrow(1)$ Let $K$ be a regular ideal of $A \bowtie I$ and let $(a, a+i) \in K$ a regular element of $A \bowtie I$. By Lemma 2.20, $a$ is a regular element of $A$ and so $\langle(a, a+i)\rangle=(a, a+i) A \bowtie I=$ $a A \bowtie(i A+a I+i I))$. Since $I=a I,\langle(a, a+i)\rangle=a A \bowtie I$. Hence $0 \times I \subseteq\langle(a, a+i)\rangle \subseteq K$ and therefore $K$ is a homogeneous ideal of $A \bowtie I$.

Remark 2.22. Let $A$ be a ring and $I$ be a proper regular ideal of $A$. Then there exists a regular ideal of $A \bowtie I$ which is not homogeneous. Indeed, if we suppose that all regular ideals of $A \bowtie I$ are homogeneous, then the ideal generated by $(c, c)$, where $c$ is a regular element in $I$, is homogeneous. By the same argument in the proof of Lemma 2.21 we show that $I=c I$ and so $c=c \lambda$ for some $\lambda \in I$ (since $c \in I$ ). So, $\lambda=1$ since $c$ is regular. Therefore, $I=A$ which is absurd since $I$ is a proper ideal of $A$.

Lemma 2.23. Let $I$ and $J$ be two ideals of a ring $A$. If $I$ and $J$ are finitely generated, then $J \bowtie I$ is a finitely generated ideal of $A \bowtie I$.

Proof. Assume $J$ is generated by a family of elements $\left\{a_{1}, a_{2} \cdots, a_{n}\right\}$ and $I$ is generated by a family of elements $\left\{k_{1}, k_{2} \cdots, k_{n}\right\}$. Let $(a, a+h) \in J \bowtie I$. Then, $a=\sum_{i=1}^{n} \alpha_{i} a_{i}$ where $\alpha_{i} \in A$ for $i=1, \cdots, n$, and since $h \in I$, then $h=\sum_{i=1}^{n} \beta_{i} k_{i}$ where $\beta_{i} \in A$ for $i=1, \cdots, n$. Hence,

$$
(a, a+h)=(a, a)+(0, h)=\sum_{i=1}^{n}\left(\alpha_{i}, \alpha_{i}\right)\left(a_{i}, a_{i}\right)+\sum_{i=1}^{n}\left(\beta_{i}, \beta_{i}\right)\left(0, k_{i}\right) .
$$

Hence $J \bowtie I$ is a finitely generated ideal of $A \bowtie I$ generated by

$$
\left\{\left(a_{i}, a_{i}\right)\right\}_{i=1, \cdots, n} \cup\left\{\left(0, k_{i}\right)\right\}_{i=1, \cdots, n} .
$$

Proof of Theorem 2.19. (1) Assume that $I$ is a flat ideal of $A$. Then $A \bowtie I$ is a faithfully flat $A$-module. Let $J$ be a regular locally principal ideal of $A$. Then $J \otimes(A \bowtie I)=$ $J(A \bowtie I)$ since $A \bowtie I$ is a faithfully flat $A$-module. So $J \otimes(A \bowtie I)=J(A \bowtie I)=J \bowtie J I$ 
by [11, Proposition 3.1(2)]. Since $J$ is regular, then $J \bowtie J I$ is a regular ideal of $A \bowtie I$. By Proposition 2.1, $J$ is a faithfully flat ideal of $A$ and so $J \otimes(A \bowtie I)=J \bowtie J I$ is a faithfully flat ideal of $(A \bowtie I)$. Thus it is locally principal by Proposition 2.1. Since $(A \bowtie I)$ is an $L P I$ ring, then $J \otimes(A \bowtie I)=J \bowtie J I$ is invertible which is equivalent to $J \otimes(A \bowtie I)$ is finitely generated. Since $A \bowtie I$ is a faithfully flat $A$-module, $J$ is a finitely generated ideal of $A$ and so invertible. Hence $A$ is an $L P I$ ring.

(2) Let $K$ be a regular locally principal ideal of $A \bowtie I$. Then $K$ is a homogeneous ideal by Lemma 2.21. So $K=J \bowtie I$ for some ideal $J$ of $A$. Let $M$ be a maximal ideal of $A$. Two cases are possible:

Case 1: $I \subseteq M$. Then $M \bowtie I$ is a maximal ideal of $A \bowtie I$ and so $J_{M} \bowtie I_{M}=(J \bowtie$ $I)_{M \bowtie I}=K_{M \bowtie I}\left(\left[12\right.\right.$, Theorem 3.8]) is a principal ideal of $(A \bowtie I)_{M \bowtie I}$. Hence $J_{M}$ is a principal ideal of $A_{M}$.

Case 2: $I \nsubseteq M$. Then $J_{M}=(J \bowtie I)_{M \bowtie I}([12$, Theorem 3.5]) is a principal ideal of $(A \bowtie I)_{M \bowtie I}$. Hence $J_{M}$ is a principal ideal of $A_{M}$.

In the both cases $J$ is locally principal. So $J$ is regular locally principal. Hence $J$ is invertible and so it is finitely generated. Thus $J \bowtie I$ is a finitely generated ideal of $A \bowtie I$ by Lemma 2.23, and therefore, $J \bowtie I$ is invertible. Hence $A \bowtie I$ is an $L P I$ ring. If furthermore $I$ is flat, then the converse follows from (1).

Example 2.24. Let $A=K \ltimes E$ where $K$ is a field and $E$ is an infinite dimensional vector space over $K$ and let $I=0 \ltimes F$ where $F$ is a finite dimensional subspace of $E$. Then :

(1) $A \bowtie I$ is an LPI ring (by Theorem 2.19(2)).

(2) $A \bowtie I$ is not Noetherian (since $A$ is not Noetherian by Example 2.18 and by $[12$, Corollary 2.9]).

\section{References}

[1] M.M. Ali, Idealization and Theorems of D.D. Anderson, Comm. Algebra 34, 44794501, 2006.

[2] M.M. Ali, Idealization and Theorems of D.D. Anderson II, Comm. Algebra 35, 27672792, 2007.

[3] D.D. Anderson and A. Mimouni, LPI domains and Pullbacks, Comm. Algebra 42, 2759-2768, 2014.

[4] D.D. Anderson and M. Roitman, A characterization of cancellation ideals, Proc. Amer. Math. Soc. 125, 2853-2854, 1997.

[5] D.D. Anderson and M. Winders, Idealization of a module, J. Commut. Algebra 1 (1), 3-56, 2009.

[6] D.D. Anderson and M. Zafrullah, Integral domains in which nonzero locally principal ideals are invertible, Comm. Algebra 39, 933-941, 2011.

[7] C. Bakkari, S. Kabbaj and N. Mahdou, Trivial extension definided by Prûfer conditions, J. Pure App. Algebra 214, 53-60, 2014.

[8] S. Bazzoni, Class semigroups of Prüfer domains, J. Algebra 184, 613-631, 1996.

[9] M. D'Anna, C.A. Finocchiaro and M. Fontana, Amalgamated algebra along an ideal, Commmutative Algebra and Applications, Walter De Gruyter, 155-172, 2009.

[10] M. D'Anna, C.A. Finocchiaro and M. Fontana, Properties of chains of prime ideals in amalgamated algebras along an ideal, J. Pure Appl. Algebra 214, 1633-1641, 2010.

[11] M. D'Anna, C.A. Finocchiaro and M. Fontana, New algebraic properties of an amalgamated algebra along an ideal, Comm. Algebra 44, 1836-1851, 2016.

[12] M. D'Anna and M. Fontana, An amalgamated duplication of a ring along an ideal: the basic properties, J. Algebra Appl. 6, 443-459, 2007.

[13] S. Glaz, Commutative coherent rings, Springer-Verlag, Lecture Notes in Mathematics, 13-71, 1989.

[14] S. Glaz and W. Vasconcelos, Flat ideals II, Manuscripta Math. 22 (4), 325-341, 1977. 
[15] F. Halter-Koch, Clifford semigroups of ideals in monoids and domains, Forum Math. 21, 1001-1020, 2009.

[16] W.C. Holland, J. Martinez, W.Wm. McGovern and M. Tesemma, Bazzoni's Conjecture, J. Algebra 320, 1764-1768, 2008.

[17] K. Hu, F.G. Wang and H. Chen, A note on LPI domains, Bull. Korean Math. Soc. 50 (3), 719-725, 2013.

[18] J.A. Huckaba, Commutative rings with zero divisors, Marcel Dekker, New YorkBasel,1988.

[19] K. Louartiti and N. Mahdou, Transfer of multiplication-like conditions in amalgamated algebra along an ideal, Afr. Diaspora J. Math. 14 (1), 119-125, 2012.

[20] G. Picozza and F. Tartarone, Flat ideals and stability in integral domains, J. Algebra 324, 1790-1802, 2010.

[21] J. Sally and W. Vasconcelos, Flat ideals I, Comm. Algebra 3 531-543, 1975.

[22] S. Xing and F.G. Wang, Two questions on domains in which locally principal ideals are invertible. J. Algebra Appl. 16 (6), 1750112, 8 pp, 2017. 